\title{
Escala de Condutas Antissociais e Delitivas: Estrutura Fatorial da Versão Portuguesa
}

\author{
Scale of Antisocial and Criminal Conducts: Factorial Structure \\ of the Portuguese Version
}

\author{
Nilton Formiga ${ }^{a}$, Vera Duarte ${ }^{b}$, Sofia Neves $^{*}, b$, Márcia Machado ${ }^{b}$ \& Francisco Machado ${ }^{b}$ \\ ${ }^{a}$ Faculdade Internacional da Paraíba, João Pessoa, PB, Brasil \\ $\&{ }^{b}$ Instituto Universitário da Maia, Maia, Portugal
}

\begin{abstract}
Resumo
A Escala de Condutas Antissociais e Delitivas tem vindo a ser usada em vários países como uma medida comportamental do desvio juvenil, evidenciando resultados muito consistentes. Este artigo apresenta e discute a análise da validade empírica da estrutura fatorial da sua versão Portuguesa. A amostra foi constituída por 443 estudantes, 305 do sexo feminino e 138 do sexo masculino, com idades compreendidas entre os 15 e os 23 anos de idade $(M=14,8 ; D P=1,90 ; M o=20)$, maioritariamente de nacionalidade portuguesa $(92,1 \%)$. Em $60,6 \%$ dos casos os/as estudantes frequentavam a universidade. Os resultados sugerem a adequação psicométrica do instrumento, corroborando a estrutura bifatorial proposta em estudos prévios. São discutidas as potencialidades da utilização do instrumento em Portugal.

Palavras-chave: Condutas antissociais e delitivas, avaliação, estrutura fatorial, Portugal.
\end{abstract}

\begin{abstract}
The Scale of Antisocial and Criminal Conducts has been used in several countries as a behavioral measure, showing very consistent results. This paper presents and discusses the analysis of the empirical validity of the factorial structure of its Portuguese version. The sample was comprised of 443 students, 305 female and 138 male, aged between 15 and 23 years $(M=14.8 ; S D=1.90 ; M o=$ 20 ), mostly Portuguese (92\%), and $60.6 \%$ were college students. Results suggest the psychometric adequacy of instrument, confirming the bi-factorial structure proposed in previous studies. Advantages of its use with the Portuguese population are discussed.
\end{abstract}

Keywords: Antisocial and criminal conducts, assessment, factorial structure, Portugal.

A delinquência juvenil afeta, de forma significativa, as sociedades atuais. Portugal não tem sido exceção. Sob o ponto de vista jurídico, a delinquência juvenil em Portugal reporta-se à prática de factos qualificados pela lei como crime, cometidos por jovens entre os 12 e os 16 anos. A este respeito, e embora a delinquência registada pela polícia tenha vindo a decrescer nos últimos anos (Ministério da Administração Interna [MAI], 2013), passando de 3880 casos registados em 2010 para 1940 registos em 2013, os resultados dos últimos estudos de delinquência juvenil auto-revelada dão conta do crescendo da chamada pequena delinquência associada à adolescência (Agra \& Castro, 2010; Cardoso, Perista, Carrilho, \& Silva, 2013; Carvalho, 2013; Carvalho \& Duarte, 2013; Pechorro et al., 2014). Em 2014 foram efetuadas 2393 participações às autoridades policiais, traduzindo um aumento de $23,4 \%$ deste tipo de criminalidade relativamente a 2013 (MAI, 2015).

Endereço para correspondência: Instituto Universitário da Maia, Avenida Carlos Oliveira - Castêlo da Maia, Avioso S. Pedro, Maia, Portugal 4475-690. E-mail: asneves@docentes.ismai.pt
Pela sua dimensão e implicações aos mais variados níveis, a delinquência juvenil tem sido objeto de estudo de diversas áreas do conhecimento, as quais têm procurado explicar as suas causas por via de caminhos teóricos-empíricos distintos e de diferentes orientações epistemo-metodológicas (Duarte \& Cunha, 2014; Duarte, Santos, Cruz, \& Grangeia, 2015; Gouveia, Santos, Pimentel, Diniz, \& Fonseca, 2009; Loeber \& Farrington, 2012; Morizot \& Kazemian, 2015; Zaluar, 2001; Zaluar \& Leal, 2001). A sua complexidade, associada aos custos pessoais, sociais e económicos, bem como a questões de coesão e segurança das sociedades (Gouveia et al., 2009) tem promovido, entre os/ as investigadores/as, difíceis debates, nem sempre unânimes. Um destes debates é reflexo da polissemia do conceito de delinquência juvenil. Sendo esta uma realidade difusa e heterogénea em si mesma, integra uma multiplicidade de manifestações que se sobrepõem, níveis de gravidade muito diferenciados e em que se entrecruzam vários tipos de fatores de risco pessoais e sociais (Le Blanc, 2008). A busca por estas e outras fronteiras conceptuais tem estado diretamente ligada aos entendimentos coletivos e às imagens construídas em torno da infância e da juventude 
Formiga, N., Duarte, V., Neves, S., Machado, M. \& Machado, F. (2015). Escala de Condutas Antissociais e Delitivas: Estrutura Fatorial da Versão Portuguesa.

(Carvalho, 2003, 2013; Carvalho \& Duarte, 2013; Duarte et al., 2015) e tem exprimido diferentes posicionamentos consoante se adote uma visão restrita do conceito de delinquência, que engloba apenas as infrações às normas jurídicas, ou se siga um olhar mais alargado, que tenta abarcar vários tipos de interdições sociais e diversos comportamentos ditos problemáticos de crianças e jovens, onde as infrações às lei são apenas uma parte (Formiga, Aguiar, \& Omar, 2008; Negreiros, 2008). Este olhar mais alargado, que perfilhamos, tem estado associado à ideia de que "a compreensão da delinquência durante a adolescência não pode ser entendida como uma oposição inqualificável entre a infração e a moral convencional ou legal" (Ferreira, 1997, p. 916), uma vez que as suas trajetórias de vida são construídas na relação entre as exigências formais da lei e as pertenças e socializações grupais.

Nesta esteira, uma das propostas com mais impacto teórico e prático tem sido a alimentada pelas perspetivas desenvolvimentais (Farrington, 1999, 2003; Loeber \& Le Blanc, 1990; Morizot \& Kazemian, 2015; Negreiros, 2008), que inclui no constructo antissocial tanto comportamentos estritamente delitivos como aqueles que, não sendo ilegais, são considerados danosos para a sociedade e para o bom desenvolvimento psicossocial do indivíduo (Loeber, Farrington, \& Petechuk, 2003). Esta questão tem particular relevância porque estas perspetivas, ao considerarem que o melhor preditor de comportamentos futuros é o comportamento passado, demonstram que é possível identificar jovens desviantes antes destes/as se tornarem ofensores/as crónicos/as. Fazem-no através de mecanismos explicativos das trajetórias delinquentes, como são a precocidade, a persistência, a duração e a variedade dos comportamentos delinquentes e os motivos subjacentes à prática (Le Blanc, 2008). Neste contexto têm vindo a ser distinguidas a delinquência ocasional, vista como uma etapa da adolescência, da delinquência persistente (Le Blanc, 2008; Moffitt, 1993). A delinquência ocasional seria aquela que se caracterizaria pela sua natureza esporádica e por um número limitado de atos delituosos de fraca gravidade, enquanto a persistente manifestar-se-ia de forma recorrente, em grau superior de gravidade, duração e diversidade, e de aparecimento precoce.

Apesar de existir hoje uma literatura consolidada sobre os fatores de risco e as trajetórias desenvolvimentais que podem levar uma criança/jovem a exibir problemas de conduta ou delinquentes (Loeber, 1990; Loeber \& Farrington, 2012), continua a não haver consenso sobre a natureza do conceito antissocial. Exemplo disso são os variados modelos teóricos e instrumentos de mensuração e avaliação da conduta antissocial que têm sido desenvolvidos nos últimos anos (Morizot \& Kazemian, 2015). Enquanto alguns modelos têm procurado explicar o desvio social dos/as jovens por referência ao diagnóstico clínico de transtornos do comportamento (Bordin \& Offord, 2000; Hare, 1996; Neumann, Hare, \& Pardini, 2014); outros explicam as diferenças individuais no comportamento de- linquente, tendo em conta a influência do contexto cultural e social mais amplo, do contexto comunitário e familiar mais próximo, das relações interpessoais e das variáveis pessoais (Andrews \& Bonta, 1994/2006; Farrington, 1999). Outros modelos ainda, procuram explicar as condutas agressivas e delitivas dos/as adolescentes e jovens através do seu sistema de crenças, atitudes e pensamento antissocial (Butler, Leschied, \& Fearon, 2007).

Uma outra proposta de mensuração do comportamento delinquente foi apresentada por Seisdedos (1988), que desenvolveu um modelo pragmático que identifica dois tipos de comportamentos socialmente desviantes: os anti-sociais e os delitivos. Estes são avaliados e medidos através do instrumento que está em análise neste texto - a Escala de Condutas Antissociais e Delitivas (ECAD). O que a conduta antissocial e delitiva parecem ter em comum é que ambas interferem nos direitos e nos deveres das pessoas, ameaçando o seu bem-estar (Formiga, 2005).

Reconhecendo a escassez de instrumentos de medida da delinquência juvenil adaptados à população portuguesa $\mathrm{e}$ considerando que a ECAD reúne características favoráveis a uma análise integrada e não restritiva do fenómeno em apreço, objetivou-se no estudo que em seguida se descreve testar a validade empírica da estrutura fatorial da versão Portuguesa da ECAD.

\section{Método}

\section{Amostra}

Fizeram parte da amostra deste estudo 443 estudantes do ensino profissional e universitário da zona norte de Portugal, 305 do sexo feminino e 138 do sexo masculino, com idades compreendidas entre os 15 e 23 anos de idade $(M=14,80 ; D P=1,90 ; M o=20)$. Os estudantes eram maioritariamente de nacionalidade portuguesa $(92,1 \%)$ e em $60,6 \%$ dos casos frequentavam o ensino universitário. A amostragem foi aleatória.

\section{Instrumentos}

Escala de Condutas Antissociais e Delitivas (ECAD, Seisdedos, 1988; Adaptado para a população brasileira por Formiga, 2003; Formiga \& Gouveia, 2003). A ECAD é uma medida comportamental composta por 40 itens, distribuídos em dois fatores: (a) condutas antissociais e (b) condutas delitivas. As condutas antissociais agregam comportamentos que desafiam a ordem social (e.g., jogar lixo no chão mesmo quando há perto um cesto de lixo; tocar a campainha na casa de alguém e sair correndo); as condutas delitivas integram comportamentos considerados como estando à margem da lei, caracterizando uma infração ou uma conduta faltosa e prejudicial a alguém ou mesmo à sociedade como um todo (e.g., roubar objetos dos carros; conseguir dinheiro ameaçando pessoas mais fracas). Para cada item, os/as respondentes devem indicar, numa escala de Likert de 0 a $9(0=$ Nunca e $9=$ Sempre $)$, a frequência do comportamento assinalado. 
No estudo de validação do instrumento para a população brasileira, desenvolvido por Formiga e Gouveia (2003), a presente escala revelou indicadores psicométricos consistentes identificando os fatores destacados acima; para as Condutas Antissociais foi encontrado um Alpha de Cronbach de 0,86 e para as Condutas Delitivas um Alpha de Cronbach de 0,92. A Análise Fatorial Confirmatória, realizada através do Lisrel 8.0, comprovou as dimensões previamente encontradas, $\chi^{2} / g l=1,35$; AGFI $=0,89$; $\mathrm{PHI}(\Phi)=0,79, p>0,05$, na análise dos principais componentes.

Ficha de Caracterização Sócio-Demográfica. O ECAD foi acompanhado de um questionário sóciodemográfico, que teve como objetivo fazer uma breve caracterização dos/as participantes deste estudo no que respeita ao sexo, idade, nacionalidade e nível de escolaridade, bem como realizar um controle estatístico de atributos que pudessem interferir diretamente nos resultados.

\section{Procedimentos}

O processo de adaptação da ECAD à população portuguesa iniciou-se com um ajustamento dos itens da versão brasileira, por forma a que a sua redação fosse mais congruente com a realidade portuguesa. Depois de refinados os itens, os mesmos foram sujeitos a um processo de reflexão falada (Almeida \& Freire, 1997) por parte de um conjunto de jovens com idades análogas às dos/as da amostra do estudo. Para se proceder à recolha dos dados foram contactados vários estabelecimentos de ensino profissional e universitário da zona norte de Portugal. Numa primeira fase solicitou-se às Direções das escolas autorização para a realização do estudo, esclarecendo-se os objetivos do mesmo e clarificando-se os critérios de inclusão: pertencer à faixa etária dos 15 aos 25 anos, não apresentar défices cognitivos severos e compreender a língua portuguesa. Os diretores de turma auxiliaram a equipa de investigação no acesso às turmas, cedendo os horários de aulas e criando uma calendarização que garantisse o envolvimento das diferentes turmas dos diferentes anos. Depois de selecionados/as os/as participantes, foram distribuídos e assinados os consentimentos informados, no caso dos menores de idade, pelos respetivos tutores legais, no caso dos maiores de 18 anos pelos próprios. A ECAD foi distribuída em contexto de sala de aula por investigadores/as com formação nos domínios da Psicologia ou Sociologia, os quais forneceram aos/às participantes as instruções necessárias ao seu preenchimento, depois de esclarecidas todas as dúvidas. $\mathrm{O}$ tempo médio de preenchimento foi de 30 minutos.

\section{Análise dos Dados}

Para a análise dos dados deste estudo utilizou-se a versão 21.0 do programa estatístico SPSS para Windows. Foram computadas estatísticas descritivas (tendência central e dispersão), Alpha de Cronbach ( $\alpha$ ), teste $t$ de Student e Análise de variância (ANOVA one-way); além destes cálculos, realizou-se uma análise fatorial confirmatória com recurso ao programa estatístico AMOS 21.0, com o intuito de se avaliar a consistência estrutural do modelo já previamente encontrado para a ECAD. Considerou-se como entrada a matriz de covariâncias, tendo sido adotado o estimador ML (Maximum Likelihood).

Uma vez que esta análise estatística é mais criteriosa e rigorosa, testou-se a estrutura teórica quanto ao modelo bifatorial. Esta análise apresenta alguns índices que permitem avaliar a qualidade de ajuste do modelo proposto (Bilich, Silva, \& Ramos, 2006; Byrne, 1989; Hair, Tatham, Anderson, \& Black, 2005; Kelloway, 1998; Tabachnick \& Fidell, 1996; Van de Vijver \& Leung, 1997). A seguir serão apresentados esses indicadores: $\mathrm{O} \chi^{2}$ (qui-quadrado) testa a probabilidade do modelo teórico se ajustar aos dados: quanto maior o valor do $\chi^{2}$ pior o ajustamento. Entretanto, ele tem sido pouco empregue na literatura, sendo mais comum considerar sua razão em relação aos graus de liberdade $\left(\chi^{2} / g l\right)$. Neste caso, valores até 3 indicam um ajustamento adequado; O Goodness-of-Fit Index (GFI) e o Adjusted Goodness-of-Fit Index (AGFI) são análogos ao $R^{2}$ na regressão múltipla e, portanto, indicam a proporção de variância-covariância nos dados explicada pelo modelo. Os valores desses indicadores variam de 0 a 1 , sendo que os valores na casa dos 0,80 e 0,90 , ou superiores, indicam um ajustamento satisfatório; A Root-Mean-Square Error of Approximation (RMSEA), com seu intervalo de confiança de 90\% (IC90\%), é considerado um indicador de "maldade" de ajuste, isto é, valores altos indicam um modelo não ajustado. Assume-se como ideal que o RMSEA se situe entre 0,05 e 0,08 , aceitando-se valores até 0,10 ; o PCLOSE trata-se de um indicador mais ponderado, o qual testa a hipótese nula do RMSEA, devendo rejeitar-se tal hipótese quando o seu valor é próximo de zero, condição essa que sugere ausência de ajuste do modelo. Com isso, recomenda-se o PCLOSE $>0,05$ como indicativo de modelo ajustado; O Comparative Fit Index (CFI) compara de forma geral o modelo estimado com o modelo nulo, considerando valores mais próximos de um como indicadores de ajustamento satisfatório; O Expected Cross-Validation Index (ECVI) e o Consistent Akaike Information Criterion (CAIC) são indicadores geralmente empregues para avaliar a adequação de um modelo determinado em relação a outro. Valores baixos do ECVI e CAIC expressam o modelo com melhor ajuste. Akaike's Information Criteria (AIC) é um critério que utiliza a parcimónia na avaliação do modelo, levando em conta o número de parâmetros estimados. É usado quando são comparados dois ou mais modelos. O modelo que apresenta melhor ajuste é o que possuiu menor AIC. Browne-Cudeck Criterion (BCC) é um critério que funciona da mesma maneira que o AIC e o CAIC com a diferença que impõe grandes penalidades para a complexidade do modelo. Bayes Information Criterion (BIC) mostra-se de forma mais consistente, uma vez que cada ajuste realizado nos componentes explicativos é gerado com base no ajuste adequado dos dados, penalizando severamente modelos com muitos parâmetros. 
Formiga, N., Duarte, V., Neves, S., Machado, M. \& Machado, F. (2015). Escala de Condutas Antissociais e Delitivas: Estrutura Fatorial da Versão Portuguesa.

\section{Resultados}

Procurando atender ao objetivo do presente estudo efetuou-se, inicialmente, uma análise dos Alphas de Cronbach ( $\alpha$; cf. Tabela 1$)$; estes apresentaram valores acima de 0,70 , seja considerando a amostra total ou as duas secções amostrais (por exemplo, N1 e N2) ou através do método Split-Half (com o coeficiente Spearman-Brown acima de 0,80 para ambas as metades, bem como o coeficiente de Guttman de 0,84). Esta condição sugere que a escala é confiável para a referida amostra, isto é, os/as participantes reconhecem as questões do instrumento a eles administrado.

Tabela 1

Indicadores Alphas de Cronbach do CAD em Jovens Portugueses

Alfa de Cronbach $(\alpha)$

\begin{tabular}{cccccc}
\hline \multirow{2}{*}{ Fatores } & $N_{\text {total }}$ & $N_{1}$ & $N_{2}$ & \multicolumn{2}{c}{ Split-Half } \\
\cline { 5 - 6 } & & & & Metade 1 & Metade2 \\
\hline CA & 0,90 & 0,91 & 0,89 & 0,82 & 0,82 \\
CD & 0,91 & 0,92 & 0,88 & 0,86 & 0,80 \\
& 0,93 & 0,94 & 0,92 & 0,88 & 0,87 \\
\hline
\end{tabular}

Notas. $\mathrm{CA}=$ Conduta Antissociais; $\mathrm{CD}=$ Condutas Delitivas; $\mathrm{CAD}=$ Condutas Desviantes $(\Sigma$ Conduta Antissociais e Condutas Delitivas); $N_{\text {total }}=$ amostra geral com 443 sujeitos; $N_{1}=$ primeira amostra com 222 sujeitos; $N_{2}=$ primeira amostra com 221 sujeitos; Split-Half $=$ Metade 1 (dez itens) e 2 (dez itens) CA, Metade 1 (dez itens) e 2 (dez itens) CD, Metade 1 (20 itens) e 2 (20 itens) CAD.

Com os Alphas acima dos exigidos pela literatura, os quais contribuíram para corroborar a medida da ECAD na amostra em análise, optou-se (acrescentando mais um elemento estatístico que salvaguardasse a confiança da medida da conduta desviante) por avaliar, a partir da análise fatorial confirmatória, a estrutura fatorial da ECAD. Empregou-se, então, o programa estatístico AMOS 21.0, testando-se o modelo estrutural proposto pelos autores supracitados (Formiga, 2003; Formiga \& Gouveia, 2003): deixando livre as covariâncias (phi, $\varphi$ ) entre os fatores da conduta antissocial e delitiva, os resultados revelaram indicadores de qualidade de ajuste para o modelo bifatorial próximos das recomendações sugeridas pela literatura estatística (Byrne, 1989; Van de Vijver \& Leung, 1997): $\chi^{2} / g l=1,87$, $\mathrm{GFI}=0,89, \mathrm{AGFI}=0,95, \mathrm{CFI}=0,96$, RMSEA $(90 \% \mathrm{IC})$ $=0,05(0,04-0,05)$, PCLOSE $=0,99$, CAIC $=2622,50 \mathrm{e}$ $\mathrm{ECVI}=3,46$; comparando tais resultados com o modelo unifatorial, $\chi^{2} / g l=4,21$, GFI $=0,69$, AGFI $=0,61$, CFI $=0,78$, RMSEA $(90 \% \mathrm{IC})=0,09(0,08-0,09)$, PCLOSE $=0,08, \mathrm{CAIC}=3913,24$ e ECVI $=7,02$, verifica-se que os melhores indicadores são os do modelo hipotetizado, previamente observados pelos autores supracitados.

Além dos indicadores referidos, outros indicadores parcimoniosos (por exemplo, o AIC, BIC e BCC), garantiram o modelo esperado, justamente por serem acompanhados do CAIC e ECVI, como um indicador comparativo da estrutura fatorial: o modelo bifatoiral - AIC $=1527,56, \mathrm{BIC}_{\text {modelobifatorial }}=2407,68$ e $\mathrm{BCC}_{\text {modelobifatorial }}=$ 1571,53 e os indicadores do modelo unifatorial - $\mathrm{AIC}_{\mathrm{mo}}$ delounifatorial $=3103,36, \mathrm{BIC}_{\text {modelounifatorial }}=3757,24 \mathrm{e} \mathrm{BCC}_{\text {mode- }}$
$=3135,88$. É preciso destacar que os indicadores parcimoniosos (por exemplo, o AIC, BIC e BCC) podem ser tomados como um indicador comparativo para verificar a melhor organização empírica dos modelos; quanto menor o valor, melhor o ajustamento, pois foi mais parciomonioso em seus ajustes de medida (Marôco, Tecedeiro, Martins, $\&$ Meireles, 2008).

Considerando os indicadores antes apresentados, destaca-se que todas as saturações (Lambdas, $\lambda$ ) tanto estiveram dentro do intervalo esperado $|0-1|$ quanto foram estatisticamente diferentes de zero $(t>1,96, p$ $<0,05$ ), denotando não haver problemas da estimação proposta da escala (cf. Tabela 2); tais resultados corroboram a existência do modelo bifatorial hipotetizado para mensurar as condutas desviantes (condutas antissociais e delitivas) dos jovens portugueses, as quais revelaram uma associação Phi $(\varphi)$ positiva entre os fatores $(\lambda=0,61)$. Desta forma, justifica-se a adequabilidade da estrutura fatorial da ECAD, de acordo com a versão proposta pelos autores Formiga (2003) e Formiga e Gouveia (2003), bem como a confiabilidade deste instrumento para a população juvenil portuguesa.

Assim sendo, observou-se que a associação Phi $(\varphi)$ entre os fatores revelou boa força associativa entre eles, bem como entre a relação item-fator (variando de 0,37 a 0,80 ), sendo esse resultado confirmado quando se realizaram as estimativas de predição, a partir da análise de regressão revelada para o modelo, ao identificar as variáveis significativas e a razão critério que estiveram dentro do que é estatisticamente exigido (cf. Tabela 3). 
Psychology/Psicologia: Reflexão e Crítica, 28(4), 718-727.

Tabela 2

Estrutura Fatorial do CAD em Jovens Portugueses

\begin{tabular}{|c|c|c|c|}
\hline$\xi$ (construto) & $\begin{array}{c}\chi \text { (variáveis) } \\
\text { [itens] }\end{array}$ & $\lambda$ & $\varepsilon$ (erros) \\
\hline \multirow{20}{*}{$\begin{array}{l}\text { Condutas } \\
\text { Antissociais }\end{array}$} & CA1 & 0,56 & 0,31 \\
\hline & $\mathrm{CA} 2$ & 0,47 & 0,22 \\
\hline & CA3 & 0,53 & 0,28 \\
\hline & CA4 & 0,54 & 0,29 \\
\hline & CA5 & 0,64 & 0,41 \\
\hline & CA6 & 0,57 & 0,33 \\
\hline & CA7 & 0,63 & 0,39 \\
\hline & CA8 & 0,55 & 0,30 \\
\hline & CA9 & 0,61 & 0,37 \\
\hline & CA10 & 0,62 & 0,38 \\
\hline & CA11 & 0,58 & 0,34 \\
\hline & CA12 & 0,55 & 0,30 \\
\hline & CA13 & 0,48 & 0,23 \\
\hline & CA14 & 0,55 & 0,30 \\
\hline & CA15 & 0,67 & 0,45 \\
\hline & CA16 & 0,65 & 0,43 \\
\hline & CA17 & 0,50 & 0,25 \\
\hline & CA18 & 0,42 & 0,18 \\
\hline & CA19 & 0,47 & 0,22 \\
\hline & CA20 & 0,57 & 0,33 \\
\hline \multirow{20}{*}{$\begin{array}{l}\text { Condutas } \\
\text { Delitivas }\end{array}$} & CD1 & 0,37 & 0,14 \\
\hline & $\mathrm{CD} 2$ & 0,68 & 0,46 \\
\hline & CD3 & 0,80 & 0,64 \\
\hline & CD4 & 0,68 & 0,46 \\
\hline & CD5 & 0,59 & 0,34 \\
\hline & CD6 & 0,52 & 0,27 \\
\hline & CD7 & 0,67 & 0,44 \\
\hline & CD8 & 0,69 & 0,48 \\
\hline & CD9 & 0,76 & 0,58 \\
\hline & CD10 & 0,68 & 0,46 \\
\hline & CD11 & 0,67 & 0,46 \\
\hline & CD12 & 0,79 & 0,63 \\
\hline & CD13 & 0,60 & 0,36 \\
\hline & CD14 & 0,76 & 0,58 \\
\hline & CD15 & 0,72 & 0,52 \\
\hline & CD16 & 0,69 & 0,47 \\
\hline & CD17 & 0,47 & 0,16 \\
\hline & CD18 & 0,45 & 0,15 \\
\hline & CD19 & 0,80 & 0,64 \\
\hline & CD20 & 0,30 & 0,09 \\
\hline
\end{tabular}

Notas. $\lambda$ Escores fatoriais da estrutura; $\varepsilon$ (erros) Erros de medida da estrutura; $\chi=$ variáveis (itens); $\xi=$ construto das Condutas Desviantes. $\mathrm{CA}=$ Condutas Antissociais; $\mathrm{CD}=$ Condutas Delitivas.
De uma forma geral, e com base nos resultados deste estudo, destaca-se que a ECAD além de fidedigna, é consistente e corrobora a estrutura bifatorial proposta previamente. Defende-se assim que reúne condições para mensurar, de forma apropriada, as condutas antissociais e delitivas dos jovens portugueses. As várias análises estatísticas realizadas para avaliação da escala apresentaram pois garantias psicométricas na amostra em questão, tendo por base diferentes evidências empíricas. Os indicadores comummente tidos em conta para corroborar o modelo pretendido (por exemplo, $\chi^{2} / g l$, GFI, AGFI, CFI, RMSEA, PCLOSE, CAIC, ECVI, AIC, BIC e BCC), bem como os Alphas de Cronbach, foram muito satisfatórios.

Reconhecida a consistência da estrutura da escala utilizada neste estudo, procurou-se avaliar a variância das respostas dos sujeitos em função do sexo e idade. Assim, em relação à idade, através de uma ANOVA one-way, observou-se que no que se refere à conduta antissocial (CAS), os jovens de 15 a 16 anos obtiveram pontuações médias superiores $\left(M=36,85 ; D P=36,09 ; \mathrm{IC}_{95 \%}-25,30\right.$ $48,39)$, quando comparados com as pontuações das demais idades, 17 a 18 anos $\left(M=31,35 ; D P=25,83 ; \mathrm{IC}_{95 \%}\right.$ 26,40-36,31), 19 a 20 anos $\left(M=25,77 ; D P=23,51 ; \mathrm{IC}_{95 \%}\right.$ -22,23-29,30), 21 a 23 anos $(M=27,33 ; D P=24,36$; $\left.\mathrm{IC}_{95 \%}-22,99-31,66 ; F_{(3,442)}=2,59, p<0,05\right)$. Apesar de resultados não serem estatisticamente significativos, importa salientar que os mais novos pontuaram mais alto na dimensão da conduta delitiva, quando comparados com jovens de idades mais avançadas. Os jovens de 15 e 16 anos, também pontuaram mais alto nas pontuações mé$\operatorname{dias}\left(M=15,07 ; D P=24,46 ; \mathrm{IC}_{95 \%}-7,25-22,90\right)$ quando comparados com outras idades, nomeadamente 17 e 18 $\operatorname{anos}\left(M=11,27 ; D P=21,52 ; \mathrm{IC}_{95 \%}-7,15-15,40\right), 19 \mathrm{e}$ 20 anos $\left(M=7,69 ; D P=14,64 ; \mathrm{IC}_{95 \%}-5,48-9,89\right)$ e 21 e $23 \operatorname{anos}\left(M=8,41 ; D P=16,64 ; \mathrm{IC}_{95 \%}-5,46-11,38 ; F\right.$ $\left.{ }_{(3,442)}=2,34, p<0,07\right)$

Ainda em relação às condutas desviantes, os jovens de 15 e 16 anos também pontuaram mais alto nas pontuações médias $\left(M=51,92 ; D P=53,38 ; \mathrm{IC}_{95 \%}-34,85-68,99\right)$ quando comparados com jovens mais velhos: 17 e 18 anos $\left(M=42,63 ; D P=41,97 ; \mathrm{IC}_{95 \%}-34,58-50,67\right), 19$ e 20 $\operatorname{anos}\left(M=33,45 ; D P=34,42 ; \mathrm{IC}_{95 \%}-28,27-36,63\right)$ e $21 \mathrm{e}$ 23 anos $\left(M=35,75 ; D P=37,77 ; \mathrm{IC}_{95 \%}-29,03-42,46 ; F\right.$ $\left.{ }_{(3,442)}=3,08, p<0,05\right)$.

Em relação ao sexo, efetuou-se um teste $t$ para amostras independentes, observando-se os seguintes resultados (cf. Tabela 4): as diferenças das pontuações médias na conduta antissocial (CAS) foram maiores para o homens $(M=$ $35,82 ; D P=30,60)$ do que para as mulheres $(M=25,37$; $D P=22,72)$. Resultados semelhantes foram observados para as condutas delitivas $\left(M_{\text {homens }}=18,28 ; D P=27,42 \mathrm{e}\right.$ $\left.M_{\text {mulheres }}=5,54 ; D P=9,80\right)$ e para as condutas desviantes $\left(M_{\text {homens }}=54,10 ; D P=52,77\right.$ e $M_{\text {mulheres }}=30,91 ; D P=$ 29,47). 
Formiga, N., Duarte, V., Neves, S., Machado, M. \& Machado, F. (2015). Escala de Condutas Antissociais e Delitivas: Estrutura Fatorial da Versão Portuguesa.

Tabela 3

Indicadores das Estimativas Preditivas entre Itens-Fatores da ECAD

\begin{tabular}{|c|c|c|c|c|c|c|}
\hline & Variáveis & & Estimativa & $D P$ & $\begin{array}{c}\text { Razão } \\
\text { Critério }\end{array}$ & $p<$ \\
\hline CAS37 & $<---$ & $\mathrm{CA}$ & 1,000 & --- & --- & --- \\
\hline CAS35 & $<---$ & $\mathrm{CA}$ & 0,483 & 0,067 & 7,263 & $*$ \\
\hline CAS34 & $<---$ & $\mathrm{CA}$ & 0,671 & 0,083 & 8,041 & $*$ \\
\hline CAS30 & $<---$ & $\mathrm{CA}$ & 1,048 & 0,115 & 9,107 & $*$ \\
\hline CAS29 & $<---$ & $\mathrm{CA}$ & 1,156 & 0,125 & 9,241 & $*$ \\
\hline CAS28 & $<---$ & $\mathrm{CA}$ & 1,104 & 0,133 & 8,285 & $*$ \\
\hline CAS26 & $<---$ & $\mathrm{CA}$ & 0,516 & 0,068 & 7,635 & $*$ \\
\hline CAS24 & $<---$ & $\mathrm{CA}$ & 0,943 & 0,104 & 9,042 & * \\
\hline CAS21 & $<---$ & $\mathrm{CA}$ & 1,098 & 0,127 & 8,651 & $*$ \\
\hline CAS18 & $<---$ & $\mathrm{CA}$ & 0,942 & 0,105 & 8,955 & $*$ \\
\hline CAS15 & $<---$ & $\mathrm{CA}$ & 1,298 & 0,147 & 8,803 & $*$ \\
\hline CAS12 & $<---$ & $\mathrm{CA}$ & 0,974 & 0,118 & 8,272 & $*$ \\
\hline CAS11 & $<---$ & $\mathrm{CA}$ & 0,710 & 0,080 & 8,903 & $*$ \\
\hline CAS10 & $<---$ & $\mathrm{CA}$ & 0,973 & 0,114 & 8,548 & $*$ \\
\hline CAS9 & $<---$ & $\mathrm{CA}$ & 1,052 & 0,117 & 8,971 & $*$ \\
\hline CAS5 & $<---$ & $\mathrm{CA}$ & 0,795 & 0,096 & 8,246 & $*$ \\
\hline CAS4 & $<---$ & $\mathrm{CA}$ & 1,220 & 0,150 & 8,111 & $*$ \\
\hline CAS02 & $<---$ & $\mathrm{CA}$ & 0,938 & 0,124 & 7,562 & * \\
\hline CAS38 & $<---$ & $\mathrm{CD}$ & 0,911 & 0,094 & 9,697 & * \\
\hline CAS01 & $<---$ & $\mathrm{CA}$ & 0,928 & 0,110 & 8,457 & * \\
\hline $\mathrm{CD} 3$ & $<---$ & $\mathrm{CD}$ & 1,000 & --- & --- & --- \\
\hline CD6 & $<---$ & $\mathrm{CD}$ & 1,013 & 0,134 & 7,567 & $*$ \\
\hline CD7 & $<---$ & $\mathrm{CD}$ & 0,974 & 0,124 & 7,872 & $*$ \\
\hline CD8 & $<---$ & $\mathrm{CD}$ & 1,293 & 0,171 & 7,577 & $*$ \\
\hline CD013 & $<---$ & $\mathrm{CD}$ & 1,088 & 0,151 & 7,193 & $*$ \\
\hline CD014 & $<---$ & $\mathrm{CD}$ & 1,228 & 0,178 & 6,897 & $*$ \\
\hline CD16 & $<---$ & $\mathrm{CD}$ & 1,232 & 0,164 & 7,492 & $*$ \\
\hline CD17 & $<---$ & $\mathrm{CD}$ & 0,906 & 0,119 & 7,606 & $*$ \\
\hline CD019 & $<---$ & $\mathrm{CD}$ & 1,205 & 0,155 & 7,773 & $*$ \\
\hline $\mathrm{CD} 20$ & $<---$ & $\mathrm{CD}$ & 1,296 & 0,172 & 7,548 & $*$ \\
\hline CD22 & $<---$ & $\mathrm{CD}$ & 0,767 & 0,102 & 7,504 & $*$ \\
\hline $\mathrm{CD} 23$ & $<---$ & $\mathrm{CD}$ & 0,933 & 0,119 & 7,847 & $*$ \\
\hline $\mathrm{CD} 25$ & $<---$ & $\mathrm{CD}$ & 0,851 & 0,117 & 7,259 & $*$ \\
\hline $\mathrm{CD} 27$ & $<---$ & $\mathrm{CD}$ & 1,407 & 0,180 & 7,798 & $*$ \\
\hline CD31 & $<---$ & $\mathrm{CD}$ & 1,105 & 0,144 & 7,662 & $*$ \\
\hline CD32 & $<---$ & $\mathrm{CD}$ & 0,917 & 0,121 & 7,563 & $*$ \\
\hline CD33 & $<---$ & $\mathrm{CD}$ & 1,134 & 0,264 & 4,290 & $*$ \\
\hline CD36 & $<---$ & $\mathrm{CD}$ & 1,064 & 0,164 & 6,487 & $*$ \\
\hline CD39 & $<---$ & $\mathrm{CD}$ & 0,951 & 0,125 & 7,624 & $*$ \\
\hline CD40 & $<---$ & $\mathrm{CD}$ & 1,100 & 0,215 & 5,128 & $*$ \\
\hline
\end{tabular}


Psychology/Psicologia: Reflexão e Crítica, 28(4), 718-727.

Tabela 4

Diferenças da Pontuação Média nas Condutas Desviantes em Função do Sexo

\begin{tabular}{lcccc}
\hline \multicolumn{1}{c}{$n$} & $M$ & $D P$ & \\
\hline CAS & & & & \\
Mulheres & 305 & 25,37 & 22,72 & $-3,56$ \\
Homens & 138 & 35,82 & 30,60 & \\
CD & & & & $-5,25$ \\
Mulheres & 305 & 5,54 & 9,80 & \\
Homens & 138 & 18,28 & 27,42 & \\
CAD & & & & $-4,79$ \\
Mulheres & 305 & 30,91 & 29,47 & 52,77 \\
Homens & 138 & 54,10 & & \\
\hline
\end{tabular}

\section{Discussão}

A partir dos resultados anteriormente descritos, comprovou-se a existência de uma relação de interdependência entre as condutas antissociais e as delitivas. Nesta ótica, uma maior pontuação das respostas do sujeito na conduta antissocial estará porventura associada a uma maior pontuação na conduta delitiva. Essa condição sugere não apenas o caráter dinâmico das condutas desviantes, mas as suas implicações sociais, psicológicas e desenvolvimentais, como discutido na introdução.

Os diversos critérios empregues na definição e comprovação do número de fatores a serem estabelecidos indicaram que a solução bifatorial é a que representa a melhor estrutura fatorial na avaliação da conduta desviante; esta estrutura revelou-se adequada ao considerar os indicadores psicométricos aceitáveis na literatura vigente $\left(\chi^{2} /\right.$ $g l$, RMR, GFI, AGFI, RMSEA, CFI, TLI, CAIC, ECVI; alpha de Cronbach); especialmente, vale a pena destacar que os indicadores parcimoniosos (por exemplo, o AIC, BIC e BCC e o PCLOSE) podem ser tomados como um indicador comparativo para verificar a melhor organização empírica dos modelos. Assim, quanto menor o valor, melhor o ajustamento. Ao comparar-se os diferentes modelos (por exemplo, unifatorial e bifatorial), observou-se que a melhor proposta empírica foi a da estrutura fatorial que se hipotetizou (Bilich et al., 2006; Byrne, 1989; Hair et al., 2005; Marôco et al., 2008).

$\mathrm{O}$ facto dos/as jovens mais novos/as da amostra terem sido aqueles/as que revelaram pontuações mais elevadas nas condutas antissociais e delitivas, divergindo dos resultados observados no estudo de Formiga (2003) e Formiga e Gouveia (2003) que mostram que os jovens mais velhos pontuam mais alto nas condutas desviantes, sugere repensar a questão da pretensa precocidade do desvio juvenil, já identificada no contexto português pelos estudos de Carva- lho $(2003,2013)$ apoiada nos estudos desenvolvimentais (Loeber \& Farrington, 2012; Thornberry \& Krohn, 2003). Se este indicador por si só não é suficiente para explicar a génese do comportamento antissocial na infância e adolescência (Lemos, 2010), tem sido constatado que a prática de delinquência precoce constitui fator de risco acrescido para o desenvolvimento de trajetórias criminais crónicas (Carvalho, 2013; Moffitt, 1993; Piquero, Farrington, \& Blumstein, 2007).

No que se refere às diferenças nas pontuações médias de homens e mulheres nas condutas antissociais e delitivas, constatou-se que os homens obtiveram maiores médias, corroborando a ideia de que a delinquência não é, como outros processos sociais, aliás, imune às influências de género (Duarte, 2015; Duarte \& Cunha, 2014). Tais resultados sugerem que os homens parecem ter uma maior propensão ao desvio social, o que pode ser justificado pelo viés cultural da instrumentalidade (Amâncio, 1994), pelas expectativas sociais em torno da masculinidade hegemónica (Connell, 2005), e por uma maior abertura a possibilidades de experiências individuais e sociais no decorrer do desenvolvimento psicossocial (Laranjeira, 2007). Estudos como os de Hunter, Figueredo, Malamuth e Becker (2004), por exemplo, apontam para uma forte relação entre a exposição dos jovens rapazes a modelos masculinos e o comportamento antissocial, a delinquência e a agressão não sexual. São várias as evidências que apontam para as diferenças de género no que respeita às práticas da delinquência juvenil. Alguns estudos sugerem mesmo haver um desfasamento substancial no nível de delinquência praticado por jovens do sexo masculino e feminino, estando na origem desta assimetria fatores como o controlo social que, no caso das jovens, tende a ser mais forte e apertado (Junger-Tas, Ribeaud, \& Cruyff, 2004). Nesta linha de pensamento têm vindo a ser desenvolvidos programas de intervenção com um enfoque de género, 
Formiga, N., Duarte, V., Neves, S., Machado, M. \& Machado, F. (2015). Escala de Condutas Antissociais e Delitivas: Estrutura Fatorial da Versão Portuguesa.

sublinhando que jovens de diferentes sexos devem ser tratados diferentemente (Duarte \& Vieites-Rodrigues, 2014; Zahn, Day, Mihalic, \& Tichavsky, 2009).

Neste contexto, fatores tais como a idade e a pertença sexual dos jovens não podem pois ser ignorados na análise do que é e de como se manifesta a delinquência juvenil.

\section{Conclusões}

De forma geral, o presente estudo procurou apresentar e discutir os resultados da análise da validade empírica da estrutura fatorial da versão Portuguesa da ECAD. Ao verificar-se a sua adequabilidade à população juvenil portuguesa, não somente se confirma a sua funcionalidade metodológica, como se comprova a sua consistência teórica, assente na díade antissocialidade-delituosidade. Assim, é possível afirmar-se que o instrumento é fidedigno, medindo os constructos a que se propõe.

A ECAD permite portanto uma compreensão da variação da conduta desviante, especialmente quando se comparam os resultados obtidos neste estudo com outros alcançados em outros países e em contextos políticos, sociais e educacionais distintos (e.g., Formiga, 2003; Formiga \& Gouveia, 2003). Sendo o seu objetivo cimeiro a avaliação quer da frequência, quer da intensidade das condutas desviantes, a sua aplicação potencia a intervenção psicossocial no sentido da prevenção dos comportamentos antissociais e delitivos, por um lado, e da sua remediação, por outro. O potencial compreensivo e preditivo do instrumento confere-lhe assim vantagens várias ao nível da sinalização de condutas problemáticas, podendo servir mesmo como um regulador do comportamento juvenil desviante. Nesta ótica, a ECAD poderá ser considerada como uma peça no quebra-cabeça dos estudos sobre delinquência (Formiga, Estevam, Camino, Mathias, \& Santos, 2010), estimulando inclusive a reflexão sobre a influência deste processo na dinâmica criminogénica da sociedade portuguesa.

Os dados do estudo que aqui se apresentou reforçam pistas importantes para o debate sobre as características do fenómeno no país. Muito embora a investigação nesta matéria esteja em franco crescimento em Portugal, o facto é que escasseiam ainda estudos orientados para a avaliação psicossocial dos/as jovens que exibem comportamentos de desvio, especialmente em grupo. Sendo esta uma característica específica da delinquência juvenil (Andrews \& Bonta, 1994/2006; Carvalho, 2013; Duarte, 2012; Le Blanc, 2008; Loeber \& Farrington, 2012), importa aprofundar as dimensões sociais (e particularmente grupais) que influenciam e condicionam o envolvimento pessoal em trajetórias desviantes. Como mostram Carvalho e Duarte (2013), é com apreensão que se vem verificando, quase um pouco por toda a Europa, que uma parte significativa da violência urbana é praticada por crianças e jovens, cada vez mais novos, tendencialmente em grupo e não raras vezes recorrendo ao uso de armas. A associação a pares desviantes situa-se, para muitas crianças e jovens, num acumular de riscos. Parece-nos pois que a aposta na caracterização destes comportamentos pode ser uma via possível de melhor compreensão das dinâmicas que lhes estão subjacentes e de desenvolvimento de estratégias de intervenção precoce. As evidências apontam no sentido da intervenção precoce ser eficaz na redução de práticas delinquentes, estando comprovado que a aplicação de sanções punitivas se revela pouco eficiente quer na inibição de condutas de natureza criminal quer na prevenção da recidiva (Carvalho, 2013; Duarte et al., 2015; Farrington, 2003)

Com a evidência da consistência fatorial do instrumento, justifica-se agora o desenvolvimento de outros estudos que afiram a convergência entre este e outros instrumentos de medida, o que não foi possível fazer-se neste trabalho e se aponta como uma limitação do mesmo. Interessante será igualmente analisar as variáveis antecedentes e consequentes da dinâmica psicossocial dos sujeitos sem história de delituosidade (e.g., intensidade no comportamento antissocial, excesso no uso e abuso de drogas lícitas e ilícitas, busca pelo sucesso e obtenção do lucro e êxito sem muito esforço, dinâmica familiar, personalidade), bem como a estrutura da ECAD noutros contextos sociais e culturais, quanto aos fatores influenciadores do desvio social e verificar a consistência dessa medida a partir de uma perspectiva desenvolvimentista e ecológica em termos da faixa etária, sexo, inserção educacional, entre outros fatores.

Cremos pois ser um modelo integrado de avaliação psicológica e social aquele que fará mais sentido na abordagem ao problema, uma vez que os fatores de risco para o desvio juvenil coabitam numa pluralidade de sistemas e de redes que se interconectam e se interinfluenciam.

\section{Referências}

Agra, C., \& Castro, J. (2010). Relatório Final do Observatório da Delinquência Juvenil. Manuscrito não publicado, Escola de Criminologia, Faculdade de Direito, Universidade do Porto, Portugal.

Almeida, L., \& Freire, T. (1997). Metodologia da investigação em Psicologia e Educação. Coimbra, Portugal: Associação dos Psicólogos Portugueses.

Amâncio, L. (1994). Masculino e feminino: A construção social da diferença. Porto, Portugal: Afrontamento.

Andrews, D. A., \& Bonta, J. (2006). The psychology of criminal conduct ( $4^{\text {th }}$ ed.). Newark, NJ: LexisNexis. (Original work published 1994)

Bilich, F., Silva, R., \& Ramos, P. (2006). Análise de flexibilidade em economia da informação: Modelagem de equações estruturais. Revista de Gestão da Tecnologia e Sistemas de Informação, 3(2), 93-122.

Bordin, I., \& Offord, D. (2000). Transtorno da conduta e comportamento anti-social. Revista Brasileira de Psiquiatria, 22(Supl. 2), 12-15. doi:10.1590/S1516-44462000000600004

Butler, S. M., Leschied, A. W., \& Fearon, P. (2007). Antisocial beliefs and attitudes in pre-adolescent and adolescent youth: The development of the antisocial beliefs and attitudes scales (ABAS). Journal of Youth Adolescence, 36(8), 1058-1071. doi:10.1007/s10964-007-9178-2

Byrne, B. M. (1989). A primer of LISREL: Basic applications and programming for confirmatory factor analytic models. New York: Springer-Verlag. 
Cardoso, A., Perista, H., Carrilho, P., \& Silva, M. (2013). Juvenile delinquency school failure and dropout in Portugal: Drafting a picture in different voices. Journal of Criminal Justice and Security, 4, 510-530.

Carvalho, M. J. (2003). Entre as malhas do desvio. Oeiras, Portugal: Celta.

Carvalho, M. J. (2013). Childhood, urban violence and territory: Children's perceptions of place and violence in public housing neighborhoods in Portugal. Children, Youth and Environments, 23(1), 124-154. doi:10.7721/chilyoutenvi.23.1.0124

Carvalho, M. J., \& Duarte, V. (2013). Crianças, jovens e a cidade: Riscos, violências e delinquências em Portugal. Latitude, $7(2), 133-166$.

Connell, R. (2005). Masculinities. Berkeley, CA: University of California Press.

Duarte, V. (2012). Discursos e percursos na delinquência juvenil feminina. Vila Nova de Famalicão, Portugal: Húmus

Duarte, V. (2015). Delinquência juvenil feminina a várias vozes. Contributos para a construção de uma tipologia de percursos transgressivos. Sociologia Problemas e Práticas, 78, 49-66. doi:10.7458/SPP2015783640

Duarte, V., \& Cunha, M. I. (2014). Violências e delinquências juvenis femininas: Aproximações à questão. In V. Duarte \& M. I. Cunha (Eds.), Violências e delinquências juvenis femininas. Género e (in)visibilidades sociais (pp. 9-24). Vila Nova de Famalicão, Portugal: Húmus.

Duarte, V., Santos, M., Cruz, O., \& Grangeia, H. (Eds.). (2015). Delinquência juvenil: Explicações e implicações. Castêlo da Maia, Portugal: Instituto Universitário da Maia.

Duarte, V., \& Vieites-Rodrigues, L. (2014). Intervenção com raparigas delinquentes: Contributos para uma discussão focada no género. In S. Gomes \& R. Granja (Eds.), Mulheres e crime - Perspetivas sobre intervenção, violência e reclusão (pp. 15-30). Vila Nova de Famalicão, Portugal: Húmus.

Farrington, D. P. (1999). Predicting persisting young offenders. Juvenile delinquency in the United States and the United Kingdom. London: St. Martin's Press.

Farrington, D. P. (2003). Developmental and life-course criminology: Key theoretical and empirical issues - The 2002 Sutherland award address. Criminology, 41(2), 221-255. doi:10.1111/j.1745-9125.2003.tb00987.x

Ferreira, P. M. (1997). Delinquência juvenil, família e escola. Análise Social, 32(143), 913-924.

Formiga, N. S. (2003). Fidedignidade da escala de condutas anti-sociais e delitivas ao contexto brasileiro. Psicologia em Estudo, 8(2), 133-138. doi:10.1590/S1413-73722003000200014

Formiga, N. S. (2005). Condutas anti-sociais e delitivas e relações familiares em duas áreas urbanas na cidade de Palmas-TO. Aletheia, 22, 63-70.

Formiga, N. S., Aguiar, M., \& Omar, A. (2008). Busca de sensação e condutas anti-sociais e delitivas em jovens. Psicologia: Ciência e Profissão, 28(4), 668-681.

Formiga, N. S., Estevam, I., Camino, C., Mathias, A., \& Santos, J. (2010). Montando o quebra-cabeça da violência entre os jovens: Testagem de um modelo teórico. Trabalho apresentado no I Congresso Internacional Adolescência e Violência: Perspectiva clínica educacional e jurídica, Brasilia, DF, Brasil.

Formiga, N. S., \& Gouveia, V. V. (2003). Adaptação e validação da escala de condutas anti-sociais e delitivas ao contexto brasileiro. Psico (Porto Alegre), 34(2), 367-388.

Gouveia, V. V., Santos, W. S., Pimentel, C. E., Diniz, P. K. C., \& Fonseca, P. N. (2009). Questionário de comportamentos anti-sociais e delitivos: Evidências psicométricas de uma versão reduzida. Psicologia: Reflexão e Crítica, 22(1), 20-28.
Hair, J. F., Tatham, R. L., Anderson, R. E., \& Black, W. (2005). Análise multivariada de dados. Porto Alegre, RS: Bookman.

Hare, R. D. (1996). Psychopathy and antisocial personality disorder: A case of diagnostic confusion. Psychiatric Times, 13(2), 39-40.

Hunter, J. A., Figueredo, A. J., Malamuth, N. M., \& Becker, J. V. (2004). Developmental pathways in youth sexual aggression and delinquency: Risk factors and mediators. Journal of Family Violence, 19(4), 233-242. doi:10.1023/ B:JOFV.0000032633.37269.1d

Junger-Tas, J., Ribeaud, D., \& Cruyff, M. (2004). Juvenile delinquency and gender. European Journal of Criminology, 1, 333-375. doi:10.1177/1477370804044007

Kelloway, K. (1998). Using LISREL for structural equation modeling: A researcher's guide. London: Sage.

Laranjeira, C. (2007). A análise psicossocial do jovem delinquente: Uma revisão da literatura. Psicologia em Estudo, 12(2), 221-227. doi:10.1590/S1413-73722007000200002

Le Blanc, M. (2008). O comportamento delinquente dos adolescentes: O seu desenvolvimento e a sua explicação. In M. Le Blanc, M. Ouimet, \& D. Szabo (Eds.), Tratado de criminologia empírica (pp. 295-336). Lisboa, Portugal: Climepsi.

Loeber, R. (1990). Development and risk factors of juvenile antisocial behavior and delinquency. Clinical Psychology Review, 10(1), 1-41. doi:10.1016/0272-7358(90)90105-J

Loeber, R., \& Farrington, D. (Eds.). (2012). From juvenile delinquency to adult crime. New York: Oxford University Press.

Loeber, R., Farrington, D., \& Petechuk, D. (2003). Child delinquency: Early intervention and prevention. Retrieved from https://www.co.ramsey.mn.us/NR/rdonlyres/82A097AE978C-4F63-9C68-30B6829A7A2F/1745/ACE_Child.pdf

Loeber, R., \& Le Blanc, M. (1990). Toward a developmental criminology. Crime and Justice, 12, 375-473. doi:10.1086/449169

Lemos, I. T. (2010). Risco psicossocial e psicopatologia em adolescentes com percurso delinquente. Análise Psicológica, 28(1), 117-132.

Marôco, J., Tecedeiro, M., Martins, P., \& Meireles, A. (2008). Estrutura fatorial de segunda ordem da Escala de Burnout de Malasch para estudantes numa amostra portuguesa. Análise Psicológica, 4(26), 639-649.

Ministério da Administração Interna. (2013). Relatório Anual de Segurança Interna. Lisboa, Portugal: Autor.

Ministério da Administração Interna. (2015). Relatório Anual de Segurança Interna. Lisboa, Portugal: Autor.

Moffitt, T. E. (1993). Adolescence-limited and life coursepersistente antissocial behavior: A development taxonomy. Psychological Review, 100, 674-701. doi:10.1037/0033295X.100.4.674

Morizot, J., \& Kazemian, L (Eds.). (2015). The development of criminal and antisocial behavior: Theory, research and practical applications. London: Springer.

Negreiros, J. (2008). Delinquências juvenis: Trajetórias, intervenção e prevenção. Porto, Portugal: Livpsic.

Neumann, C. S., Hare, R. D., \& Pardini, D. A. (2014). Antisociality and the construct of psychopathy: Data from across the globe. Journal of Personality. Advance online publication. doi:10.1111/jopy.12127

Pechorro, P., Gonçalves, R. A., Maroco, J., Gama, A. P., Neves, S., \& Nunes, C. (2014). Juvenile delinquency and psychopathic traits: An empirical study with Portuguese adolescents. International Journal of Offender Therapy and Comparative Criminology, 58(2), 174-89. doi:10.1177/0306624X12465584 
Formiga, N., Duarte, V., Neves, S., Machado, M. \& Machado, F. (2015). Escala de Condutas Antissociais e Delitivas: Estrutura Fatorial da Versão Portuguesa.

Piquero, A., Farrington, D., \& Blumstein, A. (2007). Key issues in criminal career research: New analyses of the Cambridge Study in Delinquent Development. Cambridge, UK: Cambridge University Press.

Seisdedos, N. (1988). Cuestionario A-D de conductas antisociales-delictivas. Madrid, España: TEA.

Tabachnick, B. G., \& Fidell, L. S. (1996). Using multivariate statistics. Needham Heights, MA: Allyn \& Bacon.

Thornberry, T. P., \& Krohn, M. D. (Eds.). (2003). Taking stock of delinquency. An overview of findings from contemporary longitudinal studies. New York: Kluwer Academic.

Van de Vijver, F. \& Leung, K. (1997). Methods and data analysis for cross-cultural research. Thousand Oaks, CA: Sage.

Zahn, M., Day, J., Mihalic, S., \& Tichavsky, L. (2009). Determining what works for girls in the Juvenile Justice System. Crime and Delinquency, 55(2), 266-293. doi:10.1177/0011128708330649

Zaluar, A. (2001). Violence in Rio de Janeiro: Styles of leisure, drug use, and trafficking, International Social Science Journal, 53, 369-378. doi:10.1111/1468-2451.00324

Zaluar, A., \& Leal, M. C. (2001). Violência extra e intramuros. Revista Brasileira de Ciências Sociais, 45(16), 145-164. doi:10.1590/S0102-69092001000100008 